LA CONSTRUCCIÓN DEL HOMBRE MEDIO EN JOSÉ GÓMEZ ROBLEDA: BIOTIPOLOGÍA Y MASCULINIDAD EN MÉXICO DE 1940-1960

\title{
The Construction of the Average Man in José Gómez Robleda: Biotipology and Masculinity in Mexico from 1940-1960
}

\author{
GUSTAVO ADOLFO ENRÍQUEZ GUTIÉRREZ \\ UNIVERSIDAD PEDAGÓGICA NACIONAL-MORELOS (México) \\ gusen68@hotmail.com
}

\author{
FRANCISCO RUBÉN SANDOVAL VÁZQUEZ \\ UNIVERSIDAD PEDAGÓGICA NACIONAL-MORELOS (México) \\ sandovaz@hotmail.com
}

\begin{abstract}
Resumen: el texto analiza la construcción del hombre promedio $^{1}$ como parte del proyecto político-cultural del mestizaje y la masculinidad en México de 1920 a 1940. El material se desarrolla en tres apartados: el hombre medio de 1940 a 1960; la biometría y construcción del hombre medio; y masculinidad en proletarios, deportistas, indígenas y la clase media. La biometría apareció como explicación científica sobre el cuerpo masculino con un sesgo de raza, clase y género. Asimismo, la cultura generada por los propios actores mostró nuevas corporalidades en la sociedad, así como nuevas formas de enfrentar la homogeneidad masculina dominante.
\end{abstract}

Palabras clave: biotipología, biometría, hombre medio, masculinidad

\begin{abstract}
The text analyzes the construction of average men as a part of the political-cultural project and masculinity from 1920 to 1940. The material develops in three sections: The average man from 1940 to 1960, The biometry and body outlines of the average man, and Masculinity in proletarian, sportsmen, indigenous people and middle class. Biometry appeared as a scientific explanation of the male body with a race, class and gender bias; likewise, the culture generated by the actors themselves introduced new corporalities into society, as well as new ways of facing the dominant male homogeneity.
\end{abstract}

Keywords: Average Man, Biotypology, Biometry, Masculinity

\footnotetext{
${ }^{1}$ El hombre medio fue un concepto elaborado por el investigador y funcionario José Gómez Robleda para indicar al hombre promedio o estándar cuyos rasgos físicos trató de encarnarlos en la clase media, mestiza, citadina y heterosexual. Su fin fue buscar una representación de los valores del mexicano civilizado y occidental de mediados de siglo XX. Confróntese: Imagen del mexicano de 1948.
} 


\section{Introducción}

El texto analiza el marco conceptual e interpretativo del hombre medio como parte del proyecto político-cultural del mestizaje y la masculinidad en México de 1940 a 1960. El hombre medio fue una construcción científica, política y estadística que buscó apoyar la integración y la unidad nacional de los mexicanos en esta época. José Gómez Robleda (médico-psiquiatra), en su papel de funcionario de la Secretaria de Educación Pública (SEP) e investigador del Instituto de Investigaciones Sociales (IIS) de la Universidad Nacional Autónoma de México (UNAM), estableció la mediación entre el proyecto socio-político-cultural del Estado mexicano y la intervención psicopedagógica de los gobiernos posrevolucionarios. Este funcionario académico integró en su hacer los intereses políticos del Estado a fin de apoyar la construcción del nuevo mexicano de acuerdo con los rasgos corporales de la ciencia o biotipología (Gómez y D’Aloja, 1947) y la formación de una nueva cultura nacional-mestiza (proyecto cultural mestizo y la imagen del mexicano, 1948a).

Gómez Robleda propuso una serie de estrategia para regular la conducta física, moral e intelectual del mexicano (Gómez, 1937). En palabras de Foucault (Muñiz, 2008 y Toscano, 2008), el biopoder del Estado mexicano generó una explicación del cuerpo colectivo (biopolítica) e individual (anatopolítica) de deportistas, proletarios, indígenas y la familia de clase media (Gómez, 1937, 1940, 1941, 1943, 1948b, 1949, 1959 y 1961). En este tenor, estudió el cuerpo biotipológicamente para caracterizar la salud y normalidad de la población usando medidas corporales, fisiológicas y mentales. Esta exégesis generó una serie de políticas educativas, sociales y sanitarias a fin de garantizar la higiene escolar, mental y social de los mexicanos de clase media: hombres, mestizos, modernos y civilizados (Gómez, 1946, 1948a y 1959).

De este modo, la tarea del académico y servidor público permite comprender una parte de la operación del proyecto nacional de estandarización del mexicano u hombre medio (Contreras, Flokker y Ayala, 2012; Gutmann, 1997; Schongut, 2012). Su tarea, al igual que otros académicos, políticos, investigadores y artistas del momento, fue delinear formalmente el comportamiento y sensibilidad de lo mexicano, así como su representación cultural, nacional y de género (Muñiz, 2002). El material se organiza en tres apartados: el hombre medio de 1940 a 1960; la biometría y construcción del hombre medio; y la masculinidad de proletarios, deportistas, indígenas y la clase media.

\section{El hombre medio de 1940 a 1960}

El proyecto socio-cultural de México a principios del siglo XX implicó dar un lugar a la ciudadanización del mexicano mestizo. Este proyecto se delineó en 
dos órdenes de acuerdo con Urías (2007): una vertiente antropológica que promovió la depuración racial (eugenesia) y una vertiente cultural que buscó el cambio de las mentalidades (higiene mental). La higiene mental impactó en disciplinas como la psiquiatría, la psicometría, la educación y el derecho. Por ejemplo, en el psiquiátrico de la Castañeda de la Ciudad de México, se creó y utilizó la higiene mental para evaluar la salud mental de sus internos (Maya, 2017). En la escuela, la higiene escolar llevó a la valoración de la inteligencia de los educandos (Granjas, 2009). Otra parte de este proyecto fue la educación de los anormales (Padilla, 2015), además de la educación de los indígenas y proletarios (Blanchete, 2010). Las políticas higiénicas y de salud de principios de siglo XX cobraron fuerza con la dictadura sanitaria (1917-1945) (Aréchiga, 2007). En este contexto sanitario, higiénico y médico se realizó el Primer Congreso Mexicano del Niño en 1921 y se creó el Servicio de Higiene Escolar como parte del Departamento de Salud Pública del gobierno mexicano (García y Oropeza, 2016).

Acorde con esta perspectiva higiénica, se utilizó la ciencia de este periodo como diseño de una serie de estrategias políticas y educativas a fin de formar el mestizaje nacional (Granjas, 2011 y 2009; Padilla, 2015, 2010 y 2009). El centro de esta tarea fue la creación del hombre nuevo, cuyas características se fincaron en el dominio de sí mismo a fin de apoyar la construcción de la patria y la familia. Al decir de Torres Bodet: "un tipo de hombre bondadoso, enérgico, activo, sincero, honrado, justo y emprendedor" (citado en Urías, 2007: 30). Un hombre educado, consciente de su pertenencia a la humanidad, progresista e integrado a la sana competencia internacional. $^{2}$

A pesar de este interés político, los gobiernos posrevolucionarios se encontraron con un país con carencias sociales, una industria abatida, la productividad del campo mermada, las instituciones gubernamentales decaídas por los conflictos armados y tres cuartas partes de su población viviendo en el campo (Granjas, 2011 y 2009; Suazo y López Guanzo, 2002). Esta situación condujo a un cambio en las políticas de urbanización, lo cual redujo a la población rural a $57,4 \%(14.807,534$ habitantes) y aumentó la población urbana a 42,6\% (10. 983,438 habitantes) (Canchola, 1957: 15-16). Lo anterior conformó un cambio demográfico en la población mexicana a favor de la vida urbana y sus valores. Aunado a lo anterior, los problemas de la infancia multiplicaban el diagnóstico social adverso: orfandad, mendicidad y delincuencia, además de un fuerte problema de salud: epidemias, desnutrición, alcoholismo y carencia de higiene mental (Aréchiga, 2007).

Frente a este complejo panorama social, se diseñó un enérgico programa social, cultural, político y económico. Su eje fue la construcción de una

${ }^{2}$ El hombre medio es la expresión de un nuevo humanismo que promovió el conocimiento científico del hombre a través de la economía, la sociedad y la política con el fin de establecer leyes, normas de vida, programas de Gobierno, proyectos industriales y planes educativos (Gómez, 1948: 11). Este hombre medio requería de un conocimiento científico de sus características morfológico-estructurales o somáticas, fisiológicas y psíquicas (Gómez, 1948: 13). 
ciudadanía mexicana moderna apoyada en la ciencia y en el diseño de políticas de desarrollo social, económico y cultural (Padilla, 2015, 2010 y 2009; Urías, 2007, 2005 y 2004). El trabajo académico-político partió de una base burocrática e institucional en todo el país, apuntalado por el saber científico de antropólogos, juristas, sociólogos, pedagogos, médicos y psiquiatras (Urías, 2007, 2005, 2004; Stern, 2000; Suárez y López Guazo, 2002).

Las instituciones del Estado articularon los saberes académico-políticos en tres órdenes. Primero, el saber jurídico-social a través de la "teoría de la defensa social", cuyo eje principal fue el reconocimiento del origen social de la criminalidad y la readaptación de los delincuentes. Segundo, el "saber médicopsiquiátrico" con apoyo de la eugenesia e higiene mental a fin de diseñar estrategias de mejoramiento de la salud de la población en su cuerpo, en su alma y en su conducta moral. Tercero, el saber "antropológico-educativo" mediante la gestión de una política cultural de unidad nacional, regeneración racial y mejoramiento escolar, en otras palabras, la búsqueda de la homogeneidad cultural, lingüística y social de la población (Padilla, 2015, 2010 y 2009; Stavenhagen, 2010; Urías, 2007, 2005 y 2004).

Este proyecto marcó la ciudadanía mestiza, la valía racial y la salud social como parámetros de la normalidad, vale decir, la mestizofilia ${ }^{3}$ en las políticas socio-culturales de los gobiernos posrevolucionarios como parangones de la modernidad social. La ciudadanización social implicó reconocer a la infancia como base del futuro rendimiento económico y social (Padilla, 2015, 2010 y 2009). En este sentido, la educabilidad de los mexicanos se desarrolló a través de rasgos corporales desde la masculinidad mestiza: patriótica, productiva, atlética, viril, guerrera, inteligente y sana (Gómez Robleda, 1961 y Negrete, 2016). En la femineidad mestiza prevaleció: belleza, responsabilidad, maternidad y administración del hogar y la familia (Gómez Robleda, 1959 y 1961; Muñiz, 2002).

En este contexto cultural y político, José Gómez Robleda, ${ }^{4}$ psiquiatra y funcionario público, articuló el conocimiento científico y la intervención política-cultural del Estado mexicano en sus funciones. En el Departamento de Psicopedagogía e Higiene Escolar de la SEP (Negrete, 2016), realizó el estudio sobre los escolares proletarios en 1937. Este departamento, un año antes,

\footnotetext{
${ }^{3}$ Recupero el término de mestizofilia de Alexandar Stern, quien lo toma de Agustín Basave y Claudio Lomnitz (1992), México mestizo: análisis del nacionalismo mexicano en torno a la mestizofilia de Andrés Molina Enríquez. México, Fondo Cultura Económica. Lomnitz (1992), Exits from the Labyrinth: Culture and Ideology in the Mexican National Space. Berkeley, University of California Press.

${ }^{4}$ José Gómez Robleda fue un médico y psiquiatra que trabajó en la Universidad Nacional Autónoma de México. En 1937 se desempeñó como investigador en la Secretaría de Educación Pública en el Departamento de Psicopedagogía y Médico Escolar. En 1940, el Instituto de Investigaciones Sociales de la UNAM realizó un estudio sobre las razas indígenas (la cara de los tarascos). Este investigador desarrolló, en el IIS-UNAM desde 1937 a 1962, funciones de intervención educativa, psicológica, médica e interpretativa que permitieron entender la forma en el proyecto de Estado de manera concreta a través de investigaciones sobre proletarios, deportistas, indígenas y la familia de clase media.
} 
recibió a una delegación francesa que estudió la biometría y etnografía de los otomíes. Un tiempo más tarde, en 1961, Gómez Robleda estudiaría a este grupo y lo catalogaría como representativo de la población indígena (Gómez, 1961 y Vargas, 2015). En 1941, en el contexto de la Guerra Mundial, trabajó con Lucio Mendieta, director del Instituto de Investigaciones Sociales (IIS) de la UNAM y de la Revista Mexicana de Sociología, quien tuvo vínculos con Manuel Gamio, anterior director de este instituto (Serrano, 2016). El interés de Gómez por estudiar a los grupos indígenas del país fue realizado a través del IIS-UNAM. Al mismo tiempo, en 1942 el gobierno propuso políticas en Latinoamérica desde el Instituyo Indígena Latinoamericano del que fue director Gamio (Pineda, 2012). Gómez estableció relaciones con su maestro Fernando Ocaranza, uno de los promotores de la eugenesia en México y del Congreso Mexicano del Niño de 1921, a través de su estudio sobre niños proletarios en 1937. Estos aspectos le permitieron a Gómez articularse en redes académicas, políticas y de intervención psicopedagógica.

La biotipología fue una pieza del dispositivo científico de examen, análisis e interpretación del cuerpo del mexicano usado por Gómez. Esta validación fue realizada a los escolares proletarios (1937), los criminales (1939), los deportistas (1940); los indígenas (1941, 1943, 1948b, 1949 y 1961), en las escuelas y en los docentes (1946); en la familia mexicana (1948a, 1959); y al mexicano de clases media (1962). Gómez utilizó la biotipología europea de Barbara, Kretschmer, Pende, Mac Auliffe, Sigaud y Viola en sus investigaciones. Explicó la patología constitucional de Di Tullio y Lombroso en el análisis del cuerpo del mexicano. Validó su estudio a través de los círculos académicos internacionales (Gómez, 1943). Trabajó en la Ciudad de México con el abogado Alfonzo Quiroz, la antropóloga Ada D’Aloja y formó parte del equipo de Lucio Mendieta en el Instituto de IIS-UNAM. Articuló la visión médica con la educativa desde el arquetipo occidental de ciudadanía a fin de colocar el cuerpo del mexicano en el estatus del progreso internacional. Con estos referentes teóricos explicó la calidad ciudadana del cuerpo de proletarios, criminales e indígenas.

El proletariado se volvió objeto del interés en Gómez Robleda debido a su salud, costumbres, condiciones de vida, así como a las deficiencias educativas de sus hijos. El saber médico dio cuenta de sus características físicas, anormalidades y de salud. Su hipótesis fue que la mala salud (material, social y económica) influye en el aprendizaje. Este contexto habría ocasionado patologías corporales en la salud física y moral de los escolares proletarios (Gómez Robleda, 1937).

Gómez Robleda, ${ }^{5}$ junto con el abogado Alfonso Quiroz, estudió Tendencia y ritmo de la criminalidad en México D.F. (1937). La finalidad de

\footnotetext{
${ }^{5}$ Las investigaciones de Gómez Robleda en orden cronológico fueron: escolares proletarios, 1937; tendencias criminales, 1939; deportistas, 1940; tarascos, 1941, 1943; Don Justo, 1946; biotipología, 1947; imagen del mexicano, 1948; clasificación tipológica de los grupos indígenas, 1948; zapotecas, 1949; la familia de clase media, 1959; otomíes, 1961; y psicología del mexicano, 1962.
} 
este trabajo fue identificar el aumento o disminución de la criminalidad en el Distrito Federal (actualmente Ciudad de México). Ambos investigadores analizaron la influencia del sexo, medio físico y económico. Sobre el sexo, señalaron que la criminalidad masculina era mayor que la femenina. Respecto al medio físico indicaron que tanto el calor como el frio influían en la criminalidad. El calor aumentaba los homicidios, la indisciplina carcelaria, los accesos de locura o los suicidios. Por su parte, el medio económico disminuye los delitos debido a la situación desahogada del país; sin embargo, el alcoholismo en los proletarios aumenta los delitos violentos en este sector.

En relación con los indígenas, Gómez Robleda estudió a tarascos, zapotecas y otomíes (1941, 1943, 1948b, 1949 y 1961). Desde su perspectiva, los otomíes fueron los más representativos de esta población. Ellos fueron vistos como física y mentalmente deficientes. Colocó su particularidad ciudadana corporal como deficitaria al observar su ropa, alimentación y costumbres sexuales. La caracterización psicológica de este grupo fue marcada por la exclusión social debido a un racismo de cuño histórico, reforzada por la explicación científica en boga, el desconocimiento de su lengua y la incomprensión de su cultura.

Gómez Robleda estudió a criminales, proletarios e indígenas con el objetivo de conocer el otro ángulo del cuerpo del hombre medio sustentando su investigación en juicios biológicos, fisiológicos, psiquiátricos, educativos y médicos. Se apoyó de la antropometría, la psicometría y de la biotipología para interpretar lo patológico y lo anormal. Las políticas del hombre medio operaron desde el dato estándar sin comprender el valor de otros grupos en la conformación de la nación. Una nación con múltiples oficios, trabajos y labores asentada en la pluriculturalidad, el multilingüismo y en corporeidades insumisas.

\section{La biotipología y construcción del hombre medio}

La biotipología estudió los subtipos ${ }^{6}$ corporales a través de relaciones, rasgos particulares e individuales correlacionándolos con la media social. Gómez Robleda la definió como: "un sistema vital por sí mismo, en continua reacción contra los estímulos que proceden del medio ambiente, compuesto de partes coordinadas entre sí en una unidad psicofísica indivisible que comprende todos los caracteres anatómicos y funcionales, tanto físicos como psicológicos, propios de la especie a la que pertenece" (Gómez y D’Aloja, 1947: 11).

En este tenor arrogó el cuerpo del hombre medio como un ser en el que intervino lo psicológico y lo físico. Ambos aspectos formaron la constitución

\footnotetext{
${ }^{6}$ En su estudio sobre tarascos en 1943, Gómez Robleda estableció el siguiente esquema de explicación del ser humano. Los tipos humanos fueron una categoría de personas normales. Los tipos normales fueron: tipos simples (analíticos, somáticos, funcionales, mentales y sociales), tipos mixtos (transición, somáticos, funcionales y psicosociales) y biotipos (sintéticos, biotipo de Krestschmer, Viola-Bárbara, Pende, Mac Auliffe, Sigaud y Viola). Los tipos anormales fueron: tipos patológicos y tipos criminales (Criminales de Lombroso y de Di Tullio).
} 
psicosomática de la individualidad media. La biotipología diagnosticó físicamente las aptitudes e inaptitudes tanto de los alumnos como de los trabajadores, además de la salud, deficiencias y anormalidad de los indígenas. Estos datos numéricos le permitieron a Gómez aportar una propuesta formal sobre la normalidad corporal del ciudadano mexicano de clase media (Gómez, 1937, 1947, 1959, 1961 y 1962).

El método utilizado por Gómez fue la antropometría clínica que usó instrumentos exactos, técnica especializada y máxima inmovilización del sujeto (Gómez y D’Aloja, 1947). Según Gómez y D’Aloja, el método antropométrico debió reunir las siguientes condiciones: a) las mediciones deberían de ser exactas y obtenidas con instrumentos adecuados; b) el número total de medidas del individuo debería de reducirse al mínimo; c) la selección de las medidas debería de hacerse muy escrupulosamente; d) en la elaboración de las medidas debería evitarse los cálculos complicados y largos; e) las representaciones numéricas deberían conducir a cantidades absolutas y relativas; f) la necesidad de adoptar una medida basal, g) la medida basal debería servir para la selección de los individuos y h) el método antropométrico comprendió dos partes: la representación con un mínimo de caracteres y la esquematización de la individualidad (1947: 73).

Gómez Robleda (1937) vinculó el concepto de tipo medio numérico con el de individuo normal a través de los pares salud-enfermedad, normalidadanormalidad, eficiencia-deficiencia: "Por estas razones es más justo considerar en situación extrema, al sano y al enfermo y no al normal frente al anormal: de aquí que, mientras que un individuo permanezca adaptado a la vida en sociedad y además esté capacitado para desarrollar una actividad útil a la comunidad debe considerarse como sano, independientemente de que presente algunas alteraciones" (8).

La idea de individuo normal se originó con investigaciones europeas (Kretschmer, Niceforo, Stracchey y Viola-Barbará) y del análisis estadístico de las características físicas, fisiológicas y funcionales de 500 personas de clases media de 21 a 31 años (Gómez, 1948a). Estos datos estadísticos permitieron sugerir que los miembros superiores e inferiores de los mexicanos fueron normales, pero con un tronco deficiente. Esta deficiencia somática ocasionó enfermedades infecciosas, parásitos, problemas en el aparato digestivo, dificultades respiratorias, accidentes; dificultades en oídos, ojos, reumatismo, complicaciones dermatológicas; desnutrición, nerviosismo, problemas génitourinarias: cardio-vasculares, en el embarazo, en huesos y neoplasia (Gómez, 1948a). En este sentido, el estudio de la salud morfológico-estructual del mexicano se convirtió en el eje de la interpretación y análisis de la normalidad/anormalidad.

El "tipo medio" fue: "la expresión de la adaptación secular, máxima y perfecta, de la colectividad al ambiente local y, por lo mismo, la expresión también del máximo equilibrio funcional de las partes en el todo individual" (Gómez, 1947: 18; las cursivas son mías). En otras palabras, un hombre numérico adaptado física, social e individualmente. Esta normalidad partió de 
las medidas estándares del cuerpo al comparar, por ejemplo, los músculos e inferir su media en longitud y anchura, igualdad de volumen, rendimiento máximo en velocidad y resistencia (Gómez, 1947: 46). En el caso contrario, anormalidad, caracterizó el "paramorfismo" o alternaciones del sistema muscular como la hipotonía (disminución muscular) y la hipotrofia (inferioridad de un órgano) (Gómez, 1947: 47).

De igual manera, el tipo medio corporal concretó valores como la armonía contra la desarmonía, el refinamiento contra la vulgaridad: "En algunos individuos existe una clara tendencia de armonía y refinamiento de las formas y, en otros, la tendencia contraria hacia la desarmonía vulgar. Existe sin duda, una plástica primordial hereditaria, de carácter constitucional, idealmente hermosa e idealmente "normal" en sentido estadístico, que aparece en los individuos de manera independiente al ejercicio físico" (Gómez, 1947: 54).

El hombre medio o normotipo fue: "la más sublime expresión de la belleza corresponde al estado de salud, perfección y armonía del hombre vivo" (Gómez, 1948a: 15). De este modo, el mexicano medio tuvo un carácter ligeramente intersexual: "En general, los hombres deben ser planos o anchos en vez de redondos; en consecuencia, el mexicano tiene un carácter - por este concepto- que significa intersexualidad feminoide, ciertamente, por su reducida intensidad, de muy poca importancia" (Gómez, 1948a: 20). La mexicana fue femenina, práctico-realista, predispuesta a la neurosis histérica y la locura maníaco-depresiva. Los hombres jóvenes fueron ligeramente femeninos y las mujeres tuvieron un carácter viriloide (Gómez, 1948a).

Un contraejemplo del cuerpo medio fueron los proletarios e indígenas que formaban parte de los anormales debido a deficiencias somáticas y fisiológicas producto de sus condiciones de vida (Gómez, 1937, 1941, 1943, 1948b, 1949 y 1961). La normalidad media permitió interpretar las "anomalías corporales" de los escolares proletarios e indígenas, su mala salud y caracterizar sus diferencias biotipológicas en relación con los hombres, mujeres, jóvenes y familias de clases media (Gómez, 1948b y 1959).

Esto resultados posibilitaron a Gómez Robleda sugerir mejoras en las condiciones materiales de la escuela, señaló su retraso social y la necesaria educabilidad de los indígenas. En este sentido, indicó: "Es necesario sacudir materialmente a los indios, desplazarlos de sus pueblos insalubres, sacarlos de su vida bárbaramente primitiva y monótona para romper el estado de segregación social en que viven" (Gómez, 1943: 396).

El cuerpo del hombre de clase media, del proletario y del indígena fue muestra de su mundo y condiciones de vida. La mirada del patrón de corporalidad masculina dominante manifestó una visión sobre la salud, la moralidad y el progreso, al mismo tiempo, expresó la anormalidad, la degeneración o la inferioridad constitucional producto de la pobreza nacional: "somos inferiores por pobres, no por mexicanos" (Gómez, 1948a: 72). Frente a esta problemática se propuso una serie de estudios en proletarios, deportistas, indígenas y familia de clase media, al mismo tiempo que se apoyó la 
conformación de una masculinidad y civilidad del mexicano moderno desde sus cuerpos.

\section{Masculinidades de proletarios, deportistas, indígenas y clase media}

En México, a la mitad del siglo XX y como parte de la reconstrucción posrevolucionaria, se construyó la idea del hombre medio y con ella una serie de representaciones sobre la masculinidad del cuerpo. El cuerpo masculino, de acuerdo con Gómez Robleda, permitió estudiar los distintos matices de una colectividad desde su constitución (estatura) y temperamento (peso) (Gómez, 1948b y 1949). ${ }^{7}$ La representación del cuerpo masculino justificó una visión académico-política del apoyo económico, social, atención e intervención sobre el cuerpo. Gómez Robleda reconoció el cuerpo como parte importante en el diseño de leyes, normas, programas de gobierno, proyectos económicos y educativos que coadyuvaron al crecimiento social y personal (1948a). Este marco político generó el interés por investigar el cuerpo de proletarios, deportistas, indígenas y clase media como grupos que ayudaron en la construcción de las representaciones sobre la idea de hombre medio.

Los proletarios fueron un grupo clave en el crecimiento económico, sus hijos se convirtieron en objeto de estudio a fin de apoyar este crecimiento. La escuela fue el medio formativo y educativo de estos futuros trabajadores. En este contexto, en 1937, José Gómez Robleda investigó las Características biológicas de los escolares proletarios (70 niños y 50 niñas) de la escuela primaria Ramón López Velarde, ubicada en la colonia Obrera de la Ciudad de México.

El análisis corporal se realizó en tres partes: a) consideraciones socioeconómicas; b) el problema de la edad; y c) características antropométricas (Gómez, 1937). El criterio utilizado partió de una lógica de lo general a lo particular. Analizó el contexto externo al infante seguido de su constitución fisiológica. Reconoció la marginación, la pobreza y la falta de oportunidades como aspectos que afectaron el desarrollo corporal de los infantes. En este sentido reflexionó sobre la prostitución, el alcoholismo y la religión que rodeaban a la Colonia Obrera.

La prostitución fue tachada de inmoral y como un problema de salud (Gómez, 1937). En relación con el alcoholismo, señaló: "Sin que pueda admitirse como un dato general, sí podemos decir, por informes que obtuvimos de los 'jicareros' de algunas pulquerías que las familias, sobre todo las de los obreros, consumen a cinco litros diarios cada una" (Gómez, 1937: 50). Se

\footnotetext{
${ }^{7}$ Desde la sociobiología lo masculino surge del determinismo biológico, genético y hormonal del cuerpo del hombre (Connell, 2003). La ideología moderna del cuerpo y la masculinidad dominante generó atributos estereotipados como: dominio, actividad, racionalidad, virilidad, apetito sexual desmedido, proveeduría, territorialidad y valentía desde lo público en una vida trascendente, no inmanente (Amoros, 1990 y Bordieu, 2000). Los estudios actuales de masculinidad afirman el mandato cultural, la 'desiderata', a fin de transformar al hombre en 'grandes hombres' reclamados por la sociedad (Cazés, 1998 y Godelier, 2011).
} 
indicó un estado de intoxicación permanente de los obreros por su mala alimentación, el trabajo agobiante e inmoralidad resultado de las diversiones procaces a las que recurrían. La religión la calificó como culpable de la enajenación mental de los obreros y una de las formas en que se desvirtuaron los propósitos del Estado (Gómez, 1937).

En cuanto a las escuelas señaló que presentaban una mala calidad en su construcción debido a planos defectuosos, materiales corrientes, poca ventilación, iluminación escasa además de su distribución como casa-habitación (Gómez, 1937). En referencia a las características fisiológicas-mentales de los escolares proletarios analizados, fueron evaluados como deficientes: "De una manera provisional y hechas las reservas del caso, suponemos fundadamente que puede admitirse como interpretación general que la mentalidad de los niños proletarios tiene caracteres de inferioridad" (Gómez, 1937: 235).

$\mathrm{El}$ análisis de Gómez Robleda insistió en la urgencia de intervención del Estado y en la implementación de labores de higiene mental, mejora de la infraestructura de las escuelas, disminución del trabajo infantil, incremento en la atención sanitaria, buena alimentación y buen vestido de los escolares (Gómez, 1937: 278-279). La educación de los escolares proletarios fue vista como inadecuada, sus instalaciones de mala calidad y con una inferioridad somática producto de su vida, moralidad y salud.

De este modo, la educación del hombre medio se propuso formar ciudadanos con disciplina científica, capaces de explicar los fenómenos naturales, obrar de manera racional y participar activamente en el progreso humano (Gómez, 1946 y 1962). En este sentido, el maestro fue la figura central en la ciudadanización del cuerpo del hombre medio, en palabras de Gómez Robleda: “...vive apuradamente, porque debe entregar a sus discípulos, día tras día, la última palabra del pensamiento humano” (1946: 149). La buena salud física de los infantes y la correcta disciplina funcional del maestro a través del conocimiento científico del cuerpo fueron ejes fundamentales en la formación del hombre medio (Gómez, 1962).

Al estudiar a los deportistas Gómez Robleda concluyó que presentaron intersexualidad: "viriloides en las mujeres y feminoide en los hombres" (Gómez, 1940: 337). ${ }^{8}$ Esta intersexualidad la relacionó con el híperentrenamiento y su alejamiento del trabajo productivo; destacó el deporte como técnica educativa generadora de orden y disciplina colectiva. Este último fue enfatizado como pretexto para el galanteo en los hombres; y en la mujer, para descubrir a los hombres mejor dotados para la reproducción. Señaló evitar integrar a profesionales de "sexualidad desviada"; sobre todo, impedir las tendencias "homosexuales" de quienes desarrollaban actividades deportivas (Gómez, 1940).

\footnotetext{
${ }^{8}$ Además, señaló el uso de la mano izquierda como signo de esta intersexualidad. En los tarascos indicó: "el ser lampiños, tener pelo en la frente y dientes pequeños" (1941) fueron rasgos intersexuales que favorecían la homosexualidad (1943).
} 
La idea del tipo normal medio le permitió interpretar los cuerpos de la población indígena. En 1941 y 1943, a los tarascos. En 1948, las características biotipológico de los grupos indígenas. En 1949, Zapotecas; y en 1961, Otomíes. En este periodo Gómez tipificó los rasgos físicos de los tarascos como: ascéticos, con hipotiroidismo, inhibidos, neuróticos, afeminados y bisexuales (Gómez, 1948b; Stern, 2000; y Urías, 2007, 2005). Los Zapotecos con deficiencias somáticas; fisiológicas y mentales además de una inferioridad social (1949). Los Otomíes, desde su posición, fueron representativos de la mayor parte de los pueblos indígenas cuyos rasgos físicos fueron descritos como: bajos, propensos al mareo, asmáticos, miopes, maníacos depresivos, hipersexuales, incivilizados y primitivos (Gómez, 1961). Con actitudes que expresaron la docilidad, la desconfianza, la indiferencia y poca comunicación. El punto central de su interpretación fue su inteligencia, catalogándolos como débiles mentales con una edad mental de cuatro años, deficientes en memoria y bajos en imaginación (Gómez, 1961 y Stern, 2000).

Estas ideas engrosaron la lista de atributos denigrantes sobre la población indígena y poca civilidad. Prueba de estas afirmaciones fueron las apreciaciones sobre el tipo sumario (relación entre peso y estatura) de once grupos indígenas que señaló en su estudio sobre los otomíes. En este trabajo clasificó a los grupos indígenas como más o menos deficientes (Gómez, 1961: 164-165).

El racialismo de Gómez Robleda interpretó los cuerpos de los indígenas en tipos de individuos faltos de capacidad escolar, laboral y social (Urías, 2005, 2004; Stanvenhagen, 2010; y Stern, 2000). Estos estudios valoraron a los indígenas con características biológicas deficientes, aunque señaló reiteradamente las condiciones económicas en las que vivieron y la falta de pruebas apegadas a su lengua. Lo anterior contribuyó al llenado de atributos denigrantes sobre su cuerpo, género y clase social que llevaron a la subvaloración de lo indígena en el discurso social y al racialismo en las argumentaciones científicas. En definitiva, se conforma una masculinidad inferior a la cultura de género androcéntrica y patriarcal dominante (Muñiz, 2002).

En su investigación Psicología del mexicano. Motivos de perturbación de la conducta psico-social del mexicano de clase media (1962), Gómez Robleda delineó los contornos corporales y los límites de la masculinidad del hombre medio. En una primera parte estableció un comparativo de hombres y mujeres (ver Cuadro 1). Motivos de simpatía y antipatía de enamoramiento en mujeres y hombres. Este comparativo permitió conocer el papel designado al hombre a través de los adjetivos de la mujer y viceversa.

Cuadro 1. Motivos de simpatía y antipatía de enamoramiento en mujeres y hombres.

\begin{tabular}{|l|l|}
\hline Hombre simpático a las mujeres & Mujer simpática a los hombres \\
\hline & \\
\hline
\end{tabular}




\begin{tabular}{|c|c|}
\hline Alegre, jovial, de buen humor, divertido & Hermosa \\
Inteligente & Alegre, jovial, de buen humor \\
Prudente, considerado & Inteligente \\
Amable & Afectuosa, sociable, amistosa \\
Cortés, educado & Con atractivo sexual \\
Buen conversador & Amable \\
Buen tipo & Servicial, útil \\
Interesante & Considerada \\
Generoso & Comprensiva \\
Afectuoso, amistoso & Conversadora \\
& \\
\hline
\end{tabular}

Elaboración propia a partir de José Gómez Robleda, 1962, pp. 82-83.

En resumen, estableció al hombre y la mujer simpáticos como "personas inteligentes" que divierten a otras personas (alegres, joviales de buen humor). En el caso de la mujer, además de lo anterior, tenía que "ser hermosa". El hombre también lo caracterizó como "prudente y considerado" (1962: 83). En este mismo sentido, Gómez Robleda cita a Salustiano Campo Urbano con el fin de describir el rol tradicional de la mujer como esposa y madre:

1. La mujer casada se dedicó al cuidado de la casa y de los hijos.

2. La mujer mantuvo una posición de sometimiento a su jefe y marido.

3. La mujer ansió tener muchos hijos.

4. El destino primordial fue el matrimonio.

5. La educación debió promover una excelente ama de casa, sumisa, ocupada de los hijos y dedicada al hogar.

6. El desempeño de la mujer fueron las funciones familiares dentro del hogar.

7. La mujer casada fue madre antes que esposa. (1962: 84)

En cuanto al matrimonio expresó:

1. El matrimonio fue indisoluble.

2. El fin del matrimonio fue la procreación.

3. El marido fue el proveedor del pan de la familia.

4. Las decisiones importantes fueron tomadas por el marido.

5. La religión fue el elemento fundamental de la vida familiar. (1962: 85)

A pesar de haber establecido estos roles tradicionales en el hombre y la mujer, aclaró que estos modelos provocaron perturbaciones en las familias mexicanas de clase media (1962). Lo señalado por este investigador permitió subrayar a la inteligencia como un rasgo esencial de la ciudadanía corporal de hombres y mujeres. En este sentido, señaló: “...lo más importante de los cuadros 
anteriores consiste tanto en el hombre como la mujer simpáticos deben ser personas inteligentes que divierten a los demás... la mujer simpática al hombre debe ser, además, hermosa...el hombre simpático a la mujer deber ser, también prudente y considerado" (Gómez, 1962: 85; las cursivas son mías). La inteligencia se acompañó de "belleza" en la mujer y "prudencia" en el hombre. Asimismo, añade el rol principal de la mujer como madre, esposa y subordinada a la autoridad del hombre. En el caso del hombre, proveedor, autoridad principal en el ámbito familiar y responsable de cada uno de sus miembros.

Una masculinidad asentada en la razón, en la productividad y la autoridad. Identidad masculina apoyada en la fuerza moral, económica y física equilibrada por la prudencia. Masculinidad y virilidad definida por sus contrastes con la feminidad de la madre, esposa: bella, servil, útil, considerada, comprensiva y conversadora. En esencia, un hombre inteligente que es alegre, jovial, de buen humor, divertido, prudente, considerado, amable, cortés y educado. Un hombre de buen trato y buenos modales.

El análisis de Gómez Robleda permitió la formación corporal de la ciudadanía del hombre medio en la escuela y la familia y, al mismo tiempo, promovió la institución escolar como un medio de instrucción del conocimiento científico en los futuros trabajadores y profesionistas (1946 y 1959). Una figura importante fue el maestro que lo vio como respetable y como una autoridad que inculca las razones del conocimiento científico (1959).

Ciudadanía corporal marcada por el predominio masculino, la visión urbana, la salud formada en la institución escolar enfrentada a la inferioridad de la mayor parte de la población: campesinos, indígenas y proletarios. Este médico, psiquiatra e investigador de mediados del siglo XX retrató la hegemonía corporal del ciudadano mexicano de clase media: hombre, mestizo, con rasgos occidentales, inteligente y citadino con un cuerpo medio caracterizado por ser inteligente, alegre y responsable. La mujer como: hermosa, atractiva, responsable e inteligente (1946, 1959 y 1962).

En síntesis, el hombre medio estándar, normal, fue un ciudadano con un cuerpo mestizo masculino formado por la autoridad de la institución escolar. Este hombre medio señalado por Gómez fue físicamente inteligente, usó la ciencia para el conocimiento del mundo y en su desarrollo personal (Acevedo, 2012; Gómez, 1946, 1959 y 1962; y Wilson, 2012). Este hombre requirió de un medio físico adecuado, buena alimentación y formación científica escolar conveniente a fin de integrarse como un buen trabajador, empleado o profesionista al mercado laboral (Gómez, 1937 y 1962). Criminales y deportistas contrastaron las anormalidades sociales y físicas. Los indígenas y proletarios fueron la cara discrepante de la moneda: enfermos, pobres, con rasgos difíciles de modificar, anormales, bajos y deficientes. La idea dominante de esta época fue mexicanizar al indio y civilizar al proletario a través del cuerpo occidental: inteligente, activo y promedio (Urías, 2005, 2004 y Stern, 2000). El maestro fue una figura de autoridad que formó al ciudadano 
mexicano de clase media, mestizo y occidental eliminando los rasgos proletarios e indígenas a fin de conformar al proyecto nacional imperante del cuerpo mestizo. La familia de clases media fue la imagen del hombre, la mujer y los hijos que permitieron asentar las ideas sobre la masculinidad corporal en el México moderno.

\section{Conclusiones}

Los desplazamientos conceptuales de la masculinidad corporal en criminales, deportistas, la clase media, proletarios e indígenas legitimaron los linderos sociales y los espacios del discurso científico dominante que lejos de reconocer las diferencias, las acentuó con términos técnicos psiquiátricos, sociales, jurídicos, antropológicos y educativos. Ello puso en entredicho la masculinidad del mexicano mestizo u hombre medio de mediados del siglo XX en México.

La figura de Gómez Robleda mostró la operación académico-político de los gobiernos posrevolucionarios a fin de concretar el proyecto nacional mestizo a través de diferentes estrategias de intervención social, académica, educativa y sanitaria en el cuerpo. Este burócrata académico permite entender la forma en que la ciencia, la ideológica y el interés económico se reunieron en la formación del mexicano promedio. El discurso sobre la corporalidad del mexicano promedio conformó una argumentación dominante sobre la unificación nacional, la masculinidad y la clase social mestiza al marginar el género, la clase y el origen étnico de otros grupos como obreros e indígenas.

El tipo normal o medio fue una de las directrices que sirvieron en la marcha social del mestizaje en detrimento de grupos sociales diferentes como los indígenas, los proletarios o los grupos rurales. La maquinaria institucional del hombre medio luchó contra la cultura de las comunidades indígenas y proletarias del país sin interpretar la realidad predominantemente diversa del país. El cuerpo del mexicano promedio fue un marco fuera del con-texto de la obra sobre la mexicanidad; esta corporeidad desconoció las diferentes realidades de lo mexicano en deportistas, deficientes, criminales y comunidades: rurales y/o urbano marginadas.

La biotipología fue el dispositivo científico de construcción del hombre medio apoyado de un humanismo numérico. Esta herramienta racial interpretó la estadística corporal sin comprender la historia, los valores, las creencias y las prácticas sociales asentadas en las múltiples comunidades mexicanas. Los márgenes de lo urbano, deportivo, rural, indígena y de clase media donde lo mexicano se rehízo fisuraron a estos hombres y mujeres del discurso formal del normotipo. La biotipología chocó con la filosofía hibrida, barroca de lo mexicano asentada en la cultura popular e indígena. La biotipológica fue una herramienta formal del normotipo que desconoció tradiciones, formas de vivir y portar el cuerpo mexicano de la mayoría. Voz del género que grito en la colectividad de los mexicanos, pero fue silenciada en la cultura indígena, deportiva y proletaria. 
Las investigaciones sobre criminalidad, escolares proletarios, deportistas, indígenas y clase media mostraron las otras aristas del hombre medio. Los deportistas manifestaron el nuevo alter ego de la sociedad y el cuestionamiento a la heterosexualidad. El proletario de la Ciudad de México fue una muestra de las nuevas identidades mexicanas y de otras formas de enfrentar los conflictos sociales. Los indígenas de México permitieron colocar lo mexicano en otro orden de construcción de lo masculino. El hombre medio fue criticado desde la otredad de lo indígena. La escuela y su papel en la formación de una corporalidad ciudadana masculina desde esa época hasta hoy requiere ser discutida a fin de abrir el debate al hombre y su masculinidad en el México actual.

\section{BIBLIOGRAFÍA}

AMOrós, Celia (1990), Feminismo igualdad y diferencia. México, PUEGUNAM.

ArÉCHIGA Córdoba, Ernesto (2007), "Educación, propaganda o "dictadura sanitaria". Estrategias discursivas de higiene y salubridad públicas en el México posrevolucionario, 1917-1945, en Estudios de Historia Moderna y Contemporánea de México, vol. 33, n. ${ }^{\circ} 33$, pp. 57-88.

BLANCHETTE, Thaddeus (2010), "La antropología aplicada y la administración indígena en los Estados Unidos: 1934-1945”, en Desacatos, n. 33 , pp. 3352. DOI: <https://doi.org/10.29340/33.353>.

Bourdieu, Pierre (2000), La dominación masculina, Barcelona, Anagrama.

CAZÉs, Daniel (1998), Metodología de género en los estudios de hombres, en Revista de Estudios de Género La Ventana, n. ${ }^{\circ}$ 8, pp. 100-120.

CANCHOLA, Antonio (1957), Relaciones sociales y económicas de la ciudad y el campo en México", en Revista Mexicana de Sociología, vol. XIX, n. ${ }^{\circ} 1$, pp. 15-23. DOI: <https://doi.org/ 10.2307/3537615>.

CONnell, Raewyn (2003), Masculinidades. México, PUEG-UNAM.

CONTRERAS SÁNCHEZ, Francisco; FOKKER, Benno de Keijzer y AYALAMONROY, Luis Alberto (2012), "La construcción de la masculinidad y su expresión en la sexualidad de los adolescentes" en Colecciones en salud pública, n. ${ }^{\circ}$ 8, pp. 495-518. Consultado en $<$ https://www.uv.mx/msp/files/2012/11/coleccion8FranciscoContrerasS.pdf $\geq(23 / 06 / 2018)$.

Godelier, Maurice (2011), La Producción de Grandes Hombres. Madrid, Akal. GÓmez RobledA, José (1937), Caracteristicas biológicas de los escolares proletarios. México, Departamento de Psicopedagogía y Médico Escolar/SEP.

(1940), Deportistas. México, Departamento de Psicopedagogía y Médico Escolar/Secretaría de Educación Pública. 
(1941), "La cara de los tarascos", en Revista Mexicana de Sociología, Año III, 2do Semestre, vol. III, n. ${ }^{\circ}$ 2, pp. 83-91. DOI: <https://doi.org/ $10.2307 / 3537309>$.

(1943), Pescadores y campesinos tarascos. México, Secretaría de Educación Pública, México.

(1946), Don justo. México, Secretaría de Educación Pública.

y D’ALOJA, Ada (1947), Biotipología. México, Talleres gráficos de la nación, México.

(1948a), Imagen del mexicano. México, SEP.

(1948b), "Clasificación biotipológica de los grupos indígenas de México”, en Revista Mexicana de Sociología, vol. X, n. ${ }^{\circ} 3$, pp.315-351. DOI: $<$ https://doi.org/10.2307/3537801>.

(1949), Estudio biotipológico de los zapotecas. México, Instituto de Investigaciones Sociales/ Universidad Nacional Autónoma de México.

y D’ALOJA, Ada (1959), La familia y la casa. México, Instituto de Investigaciones Sociales/ Universidad Nacional Autónoma de México.

(1961), Estudio biotipológico de los otomies. México, Instituto de Investigaciones Sociales/ Universidad Nacional Autónoma de México.

(1962), Psicología del mexicano. Motivos de perturbación de la conducta psico-social del mexicano de clase media. México, Instituto de Investigaciones Sociales/ Universidad Nacional Autónoma de México.

GutmanN, Mathew (1997), "Traficando con hombres: antropología de la masculinidad" Traducción Patricia Prieto, en Horizontes Antropológicos, Porto Alegre, Año 5, n. ${ }^{\circ}$ 10, pp. 245-286. Consultado en $<$ http://www.scielo.br/pdf/ha/v5n10/0104-7183-ha-5-10-0245.pdfs (24/07/2018). DOI: <https://doi.org/10.1590/S0104$71831999000100010>$.

GRANJAS, Josefina (2011), "El lenguaje escolar de la desigualdad en el umbral de la "primera oleada de expansión» de la enseñanza obligatoria. México en la segunda mitad del siglo XX, en Revista Mexicana de Investigación Educativa, enero-marzo, vol. 16, n. ${ }^{\circ} 48$, pp. 17-42.

(2009), "Contar y clasificar a la infancia. Las categorías de la escolarización en las escuelas primarias de la ciudad de México de 1870 a 1930", en Revista Mexicana de Investigación Educativa, vol. 14, n. ${ }^{\circ}$ 40, pp. 217-254.

MAYA GOnZÁlEZ, José Antonio (2017), "La higiene mental en el México posrevolucionario: la psiquiatría en el siglo XX”, en História, Ciências, Saúde - Manguinhos, vol. 24, n. ${ }^{\circ}$, pp. 183-185. DOI: $<$ https://doi.org/10.1590/s0104-59702017000400014>.

MuÑIZ, Elsa (2008), Cuerpo y políticas de bienestar. México en el desarrollo estabilizador (1958-1970), en Registros corporales. México, UAMAzcapotzalco, pp. 309-335.

(2002), Cuerpo, representaciones y poder. México en los albores de la reconstrucción nacional, 1920-1934. México, UAM-Azcapotzalco. 
Negrete Torres, Alejandro (2016), "El discurso medico a la práctica estatal. La labor del Departamento Psicopedagógico y Médico Escolar (19251945)", en La educación, higiene y salubridad de la infancia posrevolucionaria. México, Tesis Centro de Investigación y Docencia Económica.

PADILlA, Antonio (2015), "Estado, educación e intervención psicopedagógica en la Ciudad de México en la primera mitad del siglo XX", en Revista Mexicana de Historia de la Educación, vol. II. n. ${ }^{\circ}$ 6, pp. 207-232. DOI: $<$ https://doi.org/10.29351/rmhe.v3i6.65>.

(2010), "La educación especial en México a finales del siglo XIX y principios del XX: ideas, bosquejos y experiencias", en Revista Educación y Pedagogía, vol. 22, n. ${ }^{\circ}$ 57, mayo-agosto, p. 15-29.

(2009), "De excluidos e integrados: saberes e ideas en torno a la infancia anormal y la educación especial en México, 1920-1940”, FRENIA, vol. IX, pp. 97-134.

PinedA, Roberto (2012), El congreso indigenista de Pátzcuaro, 1940, una nueva aventura en la política indigenista en las Américas, en Baukara 2 Bitácoras de antropología e historia de la antropología en América Latina, pp. 10-28. Consultado <http://www.interindi.net/es/archivos/Baukara2 05 Pineda(10-28).pdfs (24/07/2018).

Quiroz, Alfonso; Gómez RobledA, José y Benjamín Argüelles (1939), Tendencia y ritmo de la criminalidad en México, D.F. México, DAPP.

SERrano, Margarita Olvera. (2016). "Los aportes de Lucío Mendieta y Núñez a la institucionalización de la sociología en México (1939-1951)", en Tempo Social, vol. 28, n. ${ }^{\circ} \quad 3, \quad$ pp.77-94. Consultado en

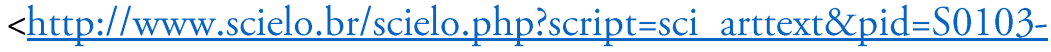
20702016000300077\&lng=en\&nrm=iso\&tlng=es> (27/07/2018). DOI: <https://doi.org/10.11606/0103-2070.ts.2016.111594>.

SuÁreZ y LóPEZ GUAZO, Laura (2009), Evolucionismo y eugenesia en México. Boletín Mexicano de Historia de Filosofía Medica, vol. 12 n. ${ }^{\circ}$ 1, pp. 19-23. Consultado en <http://www.medigraphic.com/pdfs/bmhfm/hf2009/hf091e.pdf> (26/07/2018).

(2002)? "Eugenesia, salud mental y tipología psicológica del mexicano", Asclepio, vol. LIV-2, pp.19-40. DOI: <https://doi.org/10.3989/asclepio.2002.v54.i2.139>.

STAVENHAGEN, Rodolfo (2010), "La política indigenista del Estado mexicano y los pueblos indígenas en el siglo XX". Consultado en $<$ http://www.crim.unam.mx/web/sites/default/files/2 La\%20politica indig enista.pdf $>(26 / 07 / 2018)$.

SCHONGUT Grollmus, Nicolás (2012), "La construcción social de la masculinidad: poder, hegemonía y violencia" en Psicología, Conocimiento y Sociedad, vol. 2, n. ${ }^{\circ}$ 2, pp. 27-65.

STERN, Alexandar (2000), "Mestizofilia, biotipología y eugenesia en México posrevolucionario: hacia una historia de la ciencia y el Estado, 1920-1960", en Relaciones. Estudios de historia y sociedad, vol. 81, pp. 59-91. 
Toscano López, Daniel Gihovani (2008), "EL BIO-PODER EN MICHEL FOUCAULT”, en Universitas Philosophica, vol. 25, n. ${ }^{\circ}$ 51, pp. 39-57.

URÍAS Horcasitas, Beatriz (2007), Historias secretas del racismo en México (1920-1950). México, Tusquets Editores.

(2005), "Fisiología y moral en los estudios sobre las razas mexicanas: continuidades y rupturas (siglos XIX y XX)", en Revista de indias, Vol. LXV, n. ${ }^{\circ} 234$, pp. 355-374.

(2004), "Degeneracionismo e higiene mental en el México posrevolucionario (1920-1940)", en FRENIA, vol. IV, n. 2, p. 37-67.

VARGAS DOMingueZ, Joel (2015), "Conexiones internacionales en fisiología, eugenesia y nutrición: las investigaciones sobre el metabolismo otomí en el México posrevolucionario, en Ludus Vitales, vol. XXIII", n. ${ }^{\circ} 43$, pp. 83104. Consultado en $<$ http://www.ludusvitalis.org/ojs/index.php/ludus/article/view/390/0> (24/07/2018). 